\title{
Comparison of Pain, Wound Healing, Facial Edema, and Surgeon's Comfort in Surgical Extraction of Impacted Third Molars:Surgical Scalpel Versus Radiofrequency Incision
}

\author{
N Fatahi Vanani ${ }^{1}$, A Golestaneh ${ }^{* 2}$, M MalekiGorji ${ }^{3}$ \\ 1-Dentist \\ 2- Assistant professor, department of oral and maxillofacial surgery, Dental school, Islamic Azad University, \\ Isfahan (Khorasgan) branch, Isfahan, Iran \\ 3- Postgraduate student, department of oral and maxillofacial surgery, Dental school, Islamic Azad University, \\ Isfahan (Khorasgan) branch, Isfahan, Iran
}

\begin{tabular}{|c|c|}
\hline ARTICLE INFO & ABSTRACT \\
\hline $\begin{array}{l}\text { Corresponding author: } \\
\text { Arash Golestaneh, } \\
\text { Assistant Professor, } \\
\text { Department of Oral and } \\
\text { Maxillofacial Surgery, } \\
\text { Dental School, Islamic } \\
\text { Azad University, Isfahan } \\
\text { (Khorasgan) Branch, } \\
\text { Isfahan, Iran } \\
\text { Email address: } \\
\text { drgolestaneh@gmail.com }\end{array}$ & $\begin{array}{l}\text { Background and Aim: This study aimed to compare the level of pain, wound heal- } \\
\text { ing, facial edema, and surgeon's comfort in surgical extraction of impacted third mo- } \\
\text { lars using surgical scalpel versus radiofrequency (RF) incision. } \\
\text { Materials and Methods: IThis split-mouth clinical trial evaluated } 41 \text { patients with } \\
\text { bilateral impacted third molars in one jaw with the same Pederson difficulty index } \\
\text { (between } 5 \text { and } 7 \text {, moderate difficulty). The surgical incision was made using a surgi- } \\
\text { cal scalpel on one random side and an RF device on the contralateral side. The level of } \\
\text { pain was measured using a numerical rating scale (NRS) } 7 \text { days postoperatively. The } \\
\text { wound healing was evaluated using the wound evaluation scale (WES) } 4 \text { weeks post- } \\
\text { operatively. Facial edema was quantified using a tape measure } 7 \text { days postoperatively. } \\
\text { Surgeon's comfort was assessed by asking the surgeons regarding the level of easiness } \\
\text { of the procedure. The pain score, wound healing score, facial edema, and surgeon's } \\
\text { comfort in surgical extraction of impacted third molars were compared between the } \\
\text { two sides using SPSS } 22 \text { via paired t-test and McNemar's test. } \\
\text { Result: The surgeon's comfort was significantly higher in the use of a surgical scalpel } \\
\text { (P }<0.001 \text { ). The difference in pain score (P=0.95), wound healing (P=0.32), and facial } \\
\text { edema (P }>0.05 \text { ) was not significant between the two groups. } \\
\text { Conclusion:The results of this study showed no significant difference in surgical ex- } \\
\text { traction of impacted third molars using a surgical scalpel or an RF device regarding } \\
\text { the level of pain, wound healing, or facial edema. } \\
\text { Keywords: Pain, Wound Healing, Edema, Impacted Tooth, Third Molar, Tooth Ex- } \\
\text { traction, Radiofrequency Therapy }\end{array}$ \\
\hline
\end{tabular}

\section{J Res Dent Maxillofac Sci 2020;5(2):2-6}

\section{Introduction:}

The prevalence of third molar impaction is reportedly $44.3 \%$ in Southeast Iran, ${ }^{(1)} 65.6 \%$ in the United States, ${ }^{(2)}$ and $72 \%$ in 20 - to 30 -year-olds in Sweden. ${ }^{(3)}$ Cervical caries in the distal surface of second molars, adjacent alveolar bone resorption, pain, edema, and infection are common complications associated with impacted third molars. ${ }^{(3)}$ Thus, surgical extraction of impacted third molars is often indicated.
Radiofrequency (RF) is the next generation of electrosurgery. The frequency of waves in $\mathrm{RF}$ devices has increased to 3.8-4 MHz to prevent thermal damage to the tissue and bone at the surgical site, minimize heat trauma, and enhance healing. ${ }^{(4)}$ The main advantage of $\mathrm{RF}$ devices is the induction of hemostasis immediately after the incision. Thus, it prevents hemorrhage and improves the surgeon's vision of the surgical site. The absence of bleeding is also reassuring for patients. ${ }^{(4)}$ 
Electrosurgery has some limitations; for example, contact with bone or metal should be prevented, and it is associated with excessive heat generation. However, RF devices do not have any of these limitations. ${ }^{(4)}$

Conventional impacted third molar extraction surgery involves the use of a surgical scalpel, which does not induce hemostasis right after the surgical incision. Therefore, the surgeon's vision is not excellent in conventional surgery. In addition, the use of a surgical scalpel causes fear and anxiety in many patients. ${ }^{(4)}$

At present, the use of RF for dental purposes is increasing. However, comprehensive clinical studies comparing its efficacy, advantages, and disadvantages with other conventional techniques are lacking. Also, there is controversy regarding wound healing and hemostasis following the use of RF devices. ${ }^{(5)}$

Surgical site edema is a common complication; its quantification requires ultrasound cellular imaging or a tape measure. ${ }^{(6,7)}$ Wound healing following surgical procedures can be assessed using silicon impressions, stereophotogrammetry, ultrasound, or a wound evaluation scale (WES). ${ }^{(8,9)}$ The level of pain is commonly measured using a visual analog scale (VAS), a numerical rating scale (NRS), a verbal rating scale, or pain drawing. ${ }^{(10)}$

Considering the gap of information regarding the advantages of RF in surgical extraction of impacted third molars, as well as the assumptions regarding its possible superiority to conventional scalpel surgery, this study aimed to compare the level of pain, wound healing, facial edema, and surgeon's comfort in surgical extraction of impacted third molars by conventional scalpel surgery versus RF incision.

\section{Materials and Methods:}

This split-mouth clinical trial evaluated 41 patients with bilateral impacted third molars in one jaw with the same Pederson difficulty index. (11) The patients had a mean age of $24.04 \pm 4.3$ years (ranging from 18 to 33 years). There were 21 males $(51.2 \%)$ and 20 females $(48.8 \%)$. The study has been approved by the Ethics Committee of Yazd University of Medical Sciences (IR. IAU.YAZD.REC.1398.006).

The sample size was calculated to be 41 , as- suming alpha $=0.05, \mathrm{~d}=0.05, \mathrm{z}=1.96$ at a $95 \%$ confidence interval $(\mathrm{CI})$, and the variance of the study variable to be 3.7 .

The inclusion criteria were patients with bilateral impacted third molars in one jaw with the same Pederson difficulty index (between 5 and 7 , moderate difficulty) and the American Society of Anesthesiologists Classification 1 (ASA Class I). The exclusion criteria were systemic diseases and the use of corticosteroids or other anti-inflammatory medications such as non-steroidal anti-inflammatory drugs (NSAIDS).

Surgical extraction of impacted mandibular third molars was performed using a surgical scalpel on one side and an RF device on the other side. The allocation of the technique of surgery to the side of the jaw was random and determined by flipping a coin. The same surgeon performed the surgical procedures on the two sides of each patient's jaw. A pocket flap was created by an incision at the mesial aspect of the first molar, which was extended to the distal aspect of the impaction area. Bone was removed using a round diamond bur (Teezkavan Co., Tehran, Iran) and a surgical handpiece (NSK Co., Tokyo, Japan), and the impacted third molar was extracted. The distal area next to the second molar tooth was first sutured followed by the papilla between the first and second molar teeth. Finally, the mucosa distal to the second molar was sutured using 3-0 black silk sutures (Braided Silk, Supa Co., Tehran, Iran). The sutures were removed after 7 days. The patients did not receive any medication before surgery. Analgesics, such as Ibuprofen (400mg, Raha Co., Isfahan, Iran), were prescribed every 6 hours for 3 days postoperatively. No antibiotics were prescribed.

The first follow-up session was scheduled 7 days postoperatively for suture removal, and the second session was scheduled after 4 weeks to assess wound healing.

The rate of edema was measured using a tape measure. One line was drawn from the lip corner to the tragus with another line from the tragus to the pogonion (Figure 1). The sum of these two lines was calculated before surgery (the baseline) and 7 days postoperatively. ${ }^{(7)}$ 


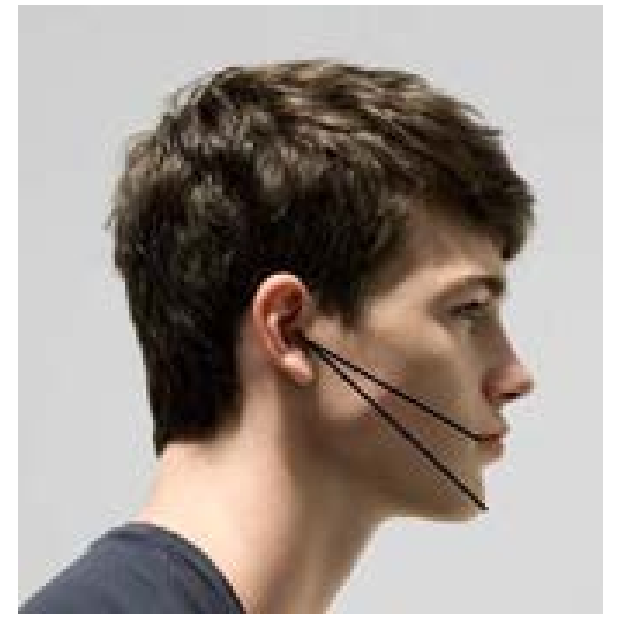

Figure 1. Lines from the lip corner to the tragus and from the tragus to the pogonion

A checklist was designed to assess the surgeon's comfort in the conduction of the surgical procedures.

The patients were requested to express their level of pain using a 0-10 NRS. Score 0 indicated no pain at all, scores 1-3 indicated mild pain, scores 4-6 indicated moderate pain, scores 7-9 indicated severe pain, and score 10 indicated the worst pain imaginable. ${ }^{(12)}$ The mean scores were then calculated.

Wound healing was evaluated and compared using the WES, which evaluates 6 clinical aspects, namely step-off margins, contour abnormality, scar width greater than $2 \mathrm{~mm}$, edge inversion, edema, and overall cosmesis. Score 0 was given for the presence and score 1 was given for the absence of any of the above-mentioned symptoms. The total score was the sum of all the scores. ${ }^{(12)}$

Data were analyzed using descriptive and inferential statistics via SPSS version 22 (SPSS Inc., Chicago, IL, USA). The KolmogorovSmirnov test was applied to assess the normal distribution of data. The mean and standard deviation (SD) of the variables were reported, and data were analyzed using paired t-test and McNemar's test at the significance level of 0.05 .

\section{Results:}

Of all, 27 surgeons (65.9\%) reported higher comfort in the use of a surgical scalpel, and 14 surgeons (34.1\%) reported higher comfort in the use of an RF device. According to McNemar's test, this difference was statistically significant $(\mathrm{P}<0.001)$.

The Kolmogorov-Smirnov test showed that the pain scores according to the NRS, the wound healing scores according to the WES, and the rate of facial edema on the two sides were all normally distributed. Thus, these parameters were compared between the two groups using a parametric paired t-test.

Table 1 shows the measures of central dispersion for pain scores according to the NRS and wound healing scores according to the WAS in the two groups. According to the paired t-test, the mean pain scores $(\mathrm{P}=0.95)$ and the mean wound healing scores $(\mathrm{P}=0.32)$ were not significantly different between the two sides. In other words, the technique of surgery had no significant effect on the pain or wound healing scores one week postoperatively $(\mathrm{P}>0.05)$.

Table 1. Measures of central dispersion for pain scores according to the numerical rating scale (NRS) and wound healing scores according to the wound evaluation scale (WES) in the two groups

\begin{tabular}{|c|c|c|c|c|c|}
\hline \multirow{2}{*}{ Score } & \multicolumn{2}{|c|}{ Radiofrequency } & \multicolumn{2}{|c|}{ Scalpel } & \multirow{2}{*}{ P-value } \\
\hline & Mean & SD & Mean & SD & \\
\hline Pain (NRS) & 5.3 & 2.5 & 5.4 & 2.2 & 0.95 \\
\hline Wound healing (WES) & 5 & 0.2 & 4.9 & 0.3 & 0.32 \\
\hline
\end{tabular}

\section{SD=Standard Deviation}

Table 2 shows the mean tape measurements (indicative of facial edema) at the baseline and one week postoperatively in the two groups. The paired t-test showed that the mean tape measurements were not significantly different between the two groups at the baseline $(\mathrm{P}=0.10)$ or one week postoperatively $(\mathrm{P}=0.14)$. In other words, the technique of surgery had no significant effect on facial edema. The mean change in tape measurements, one week postoperatively compared to the baseline, was $0.7 \pm 0.5$ in the RF group and $0.8 \pm 0.6$ in the scalpel group with no significant difference $(\mathrm{P}=0.50)$. In other words, the technique of surgery had no significant effect on the change in tape measurements. 
Table 2. Mean tape measurements (indicative of facial edema) at the baseline and one week postoperatively in the two groups

\begin{tabular}{cccccc}
\hline \multirow{2}{*}{ Time } & \multicolumn{3}{c}{ Radiofrequency } & \multicolumn{2}{c}{ Scalpel } \\
\cline { 2 - 5 } & Mean & SD & Mean & SD & P-value \\
\hline Baseline & 17.6 & 1.5 & 17.7 & 1.6 & 0.10 \\
\hline One week postoperatively & 18.3 & 1.5 & 18.5 & 1.5 & 0.14 \\
\hline
\end{tabular}

SD=Standard Deviation

\section{Discussion:}

This study compared the level of pain, wound healing, facial edema, and surgeon's comfort in surgical extraction of impacted third molars by conventional scalpel surgery versus RF incision. RF waves, as applied in medicine, cause thermal ablation of a defined volume of tissue. The RF ablation electrode (the cathode) is closed using dispersing pads on the patient's thighs. ${ }^{(4)}$

There is a very high-energy flux around the electrode tip because of its small cross-sectional area. Therefore, tissue damage is limited to the circuit surrounding the electrode tip. This study showed that the surgeon's comfort was significantly higher in the use of a surgical scalpel $(\mathrm{P}<0.001)$. This finding may be due to inadequate knowledge of surgeons about the correct use of RF devices. ${ }^{(4)}$

The difference in pain $(\mathrm{P}=0.95)$ and wound healing $(\mathrm{P}=0.32)$ scores was not significant between the two groups. Sherman reported that excellent hemostasis can be achieved by the use of RF with optimal wavelength and electrode. ${ }^{(4)}$

These two parameters can lead to minimal edema and excellent healing. Furthermore, RF allows faster incision and better visualization of the surgical site, which would result in shorter surgical time. RF is safe and cost-effective as well. On the other hand, the use of a surgical scalpel can cause scarring at the surgical site and enhance microbial contamination. The correct use of RF devices would eliminate these shortcomings. (4) In our study, no significant difference was noted in facial edema between the two groups. Moreover, mean tape measurements did not change over time.

Silverman et al ${ }^{(13)}$ compared RF, carbon dioxide (CO2) laser, and monopolar electrosurgery and concluded that RF was superior to all other techniques due to minimal damage to tissue, which was different from our findings since we found no significant difference between the two techniques in terms of pain score or wound healing. Hasar et al evaluated the effect of a scalpel, electrosurgery, and RF on pain, hemostasis, and wound healing in rats. ${ }^{(14)}$ They reported that weight loss in rats in the first 7 days postoperatively was lower in the scalpel group; thus, this group had a lower level of pain than the electrosurgery and RF groups. However, the rats finally returned to their baseline weight, and the difference did not reach a statistical significance. The level of edema was also the same in the three groups. ${ }^{(14)}$

Their results were in line with our findings. Kim et al compared RF with conventional scalpel surgery and concluded that RF had a high thermal effect on trigeminal neuralgia. ${ }^{(15)}$

Our results were different from their findings, which may be due to the difference in the target site and the type of procedure. Bovaira et al evaluated the efficacy of treatment with RF in five patients with trigeminal neuralgia and concluded that RF was more effective than pharmaceutical therapy for this purpose. ${ }^{(16)}$ Our results were different from the findings of Bovaira et al, ${ }^{(16)}$ which may be due to the difference in the site of treatment and the type of procedure. Kashkouli et al evaluated the efficacy of RF for upper blepharoplasty in comparison with a surgical scalpel and reported that RF caused deeper tissue damage. ${ }^{(17)}$ Our results were in line with theirs despite the differences in the type of procedure and the surgical site.

Future studies with larger sample sizes are required to compare surgical scalpels, RF, and electrosurgery to find accurate results in this respect.

\section{Conclusion:}

The results of this study showed no significant difference in surgical extraction of impacted third molars with conventional scalpel surgery and RF incision regarding the level of pain, wound healing, and facial edema.

\section{References}

1. Hashemipour MA, Tahmasbi-Arashlow M, FahimiHanzaei F. Incidence of Impacted Mandibular and Maxillary Third Molars: A Radiographic Study in a 
Southeast Iran Population. Med Oral Patol Oral Cir Bucal. 2013 Jan 1;18(1):e140-5.

2. Kanneppady SK, Balamanikandasrinivasan, Kumaresan R, Sakri SB. A comparative study on radiographic analysis of impacted third molars among three ethnic groups of patients attending AIMST Dental Institute, Malaysia. Dent Res J (Isfahan). 2013 May;10(3):353-8.

3. Passi D, Singh G, Dutta S, Srivastava D, Chandra L, Mishra S, et al. Study of pattern and prevalence of mandibular impacted third molar among Delhi-National Capital Region population with newer proposed classification of mandibular impacted third molar: A retrospective study. Natl J Maxillofac Surg. 2019 JanJun;10(1):59-67.

4. Sherman JA. Oral surgery simplified with radiosurgery. Dent Today. 2008 Feb;27(2):123-4.

5. Sumit S, Simarpreet S, Gurminder S, Vikas S. Radiosurgery in Dentistry - A Brief. Ann Dent Res. 2012;2(1):8-20.

6. Machtaler S, Knieling F, Luong R, Tian L, Willmann JK. Assessment of inflammation in an acute on chronic model of inflammatory bowel disease with ultrasound molecular imaging. Theranostics. 2015 Aug 8;5(11):1175-86.

7. Grossi GB, Maiorana C, Garramone RA, Borgonovo A, Beretta M, Farronato D, et al. Effect of submucosal injection of dexamethasone on postoperative discomfort after third molar surgery: a prospective study. J Oral Maxillofac Surg. 2007 Nov;65(11):2218-26.

8. Humbert PG, Meaune S, Gharbi T. Wound healing assessment. Phlebolymphology. 2004 Spring;47:312-19. 9. Hamdan S, Pastar I, Drakulich S, Dikici E, Tomic-Canic M, Deo S, et al. Nanotechnology-Driven Therapeutic Interventions in Wound Healing: Potential Uses and Applications. ACS Cent Sci. 2017 Mar 22;3(3):163-75.

10. Haefeli M, Elfering A. Pain assessment. Eur Spine J. 2006 Jan;15 Suppl 1:S17-24.

11. Zhang Y, Ruan Z, Shen M, Tan L, Huang W, Wang $\mathrm{L}$, et al. Clinical effect of platelet-rich fibrin on the preservation of the alveolar ridge following tooth extraction. Exp Ther Med. 2018 Mar;15(3):2277-86.

12. Dijkers M. Comparing quantification of pain severity by verbal rating and numeric rating scales. $J$ Spinal Cord Med. 2010 Jun;33(3):232-42.

13. Silverman EB, Read RW, Boyle CR, Cooper R, Miller WW, McLaughlin RM. Histologic comparison of canine skin biopsies collected using monopolar electrosurgery, $\mathrm{CO} 2$ laser, radiowave radiosurgery, skin biopsy punch, and scalpel. Vet Surg. 2007 Jan;36(1):50-6.

14. Hasar ZB, Ozmeric N, Ozdemir B, Gökmenoğlu C, Baris E, Altan G, et al. Comparison of radiofrequency and electrocautery with conventional scalpel incisions.
J Oral Maxillofac Surg. 2016 Nov 1;74(11):2136-41. 15. Kim JH, Yu HY, Park SY, Lee SC, Kim YC. Pulsed and conventional radiofrequency treatment: which is effective for dental procedure-related symptomatic trigeminal neuralgia? Pain Med. 2013 Mar;14(3):4305.

16. Bovaira $M$, Peñarrocha $M$, Peñarrocha $M$, Calvo A. Conventional radiofrequency treatment in five patients with trigeminal neuralgia. Med Oral Patol Oral Cir Bucal. 2013 Jan 1;18(1):e76-80.

17. Kashkouli MB, Kaghazkanai R, Mirzaie AZ, Hashemi M, Parvaresh MM, Sasanii L. Clinicopathologic comparison of radiofrequency versus scalpel incision for upper blepharoplasty. Ophthalmic Plast Reconstr Surg. 2008 Nov-Dec;24(6):450-3.

18. Etemadi A, Bitaraf T, Amini A, Goudarzi M, Nadafpour N. Bacterial Accumulation on TriclosanCoated and Silk Sutures After Dental Implant Surgery. J Res Dentomaxillofac Sci. 2019;4(3):1-4. 